\title{
Using the Variable Pump Intensity method to measure optical gains and unveil photophysical and photonic phenomena in active waveguides
}

\author{
Luis Cerdán, ${ }^{1 *}$ Marco Anni, ${ }^{2}$ Maria Luisa De Giorgi, ${ }^{2}$ Pedro G. Boj, ${ }^{3}$ María Ángeles Díaz-García ${ }^{4}$ \\ 1. Instituto de Química Física "Rocasolano”, Consejo Superior de Investigaciones Científicas (CSIC), C/ Serrano 119, 28006, Madrid, \\ Spain (Affiliation until 2017).e-mail: lcerdanphd@gmail.com \\ 2. Dipartimento di Matematica e Fisica "Ennio de Giorgi”, Universitá del Salento, via per Arnesano, 73100, Lecce, Italy \\ 3. Departamento de Óptica, Farmacología y Anatomía, Instituto Universitario de Materiales de Alicante y Unidad Asociada UA-CSIC, \\ Universidad de Alicante, 03080, Alicante, Spain \\ 4. Departamento de Física Aplicada, Instituto Universitario de Materiales de Alicante y Unidad Asociada UA-CSIC, Universidad de \\ Alicante, 03080, Alicante, Spain
}

The last decades have witnessed the advent of a myriad of new solution-processed laser materials that can be implemented as low-cost, flexible, and/or integrated waveguide devices [1]. This profusion makes necessary the development of reliable methods to delineate all their amplifying signatures and thus to open the door to appropriate cross-sample comparisons. In this sense, the generation of Amplified Spontaneous Emission (ASE) is routinely used to assess the performance of active materials prior to developing thin film lasers. In particular, the Variable Stripe Length (VSL) method has been profusely used for decades to measure the optical gain in these materials and devices, but experimental and theoretical evidences cast doubts upon its reliability [2]. Seeking to solve those problems, a new formalism to fully characterize the amplification properties of active waveguides based on the most common Variable Pump Intensity (VPI) method has been recently reported by one of us [3]. The analytical expression describing the growth of ASE intensity $I_{A S E}\left(\lambda, I_{p}, L\right)$ as a function of the wavelength, pump value $\left(I_{p} ;\right.$ energy/density/intensity/flux/...) and stripe length $(L)$ reads [3]:

$$
I_{A S E}\left(\lambda, I_{p}, L\right)=\frac{\Omega(\lambda) \eta\left(I_{p}\right)}{\gamma(\lambda) \Delta n\left(I_{p}\right)-\alpha(\lambda)}\left(e^{\left(\gamma(\lambda) \Delta n\left(I_{p}\right)-\alpha(\lambda)\right) L}-1\right),
$$

with the population inversion $\Delta n$ following a well-defined but cumbersome rational function. It was shown that eq. (1) allows retrieving from the ASE spectra of a single VPI experiment, the spectrally resolved losses coefficient $\alpha(\lambda)$ and net optical gains $g=\gamma \Delta n-\alpha(\lambda)$ at all pump values (Fig. 1).
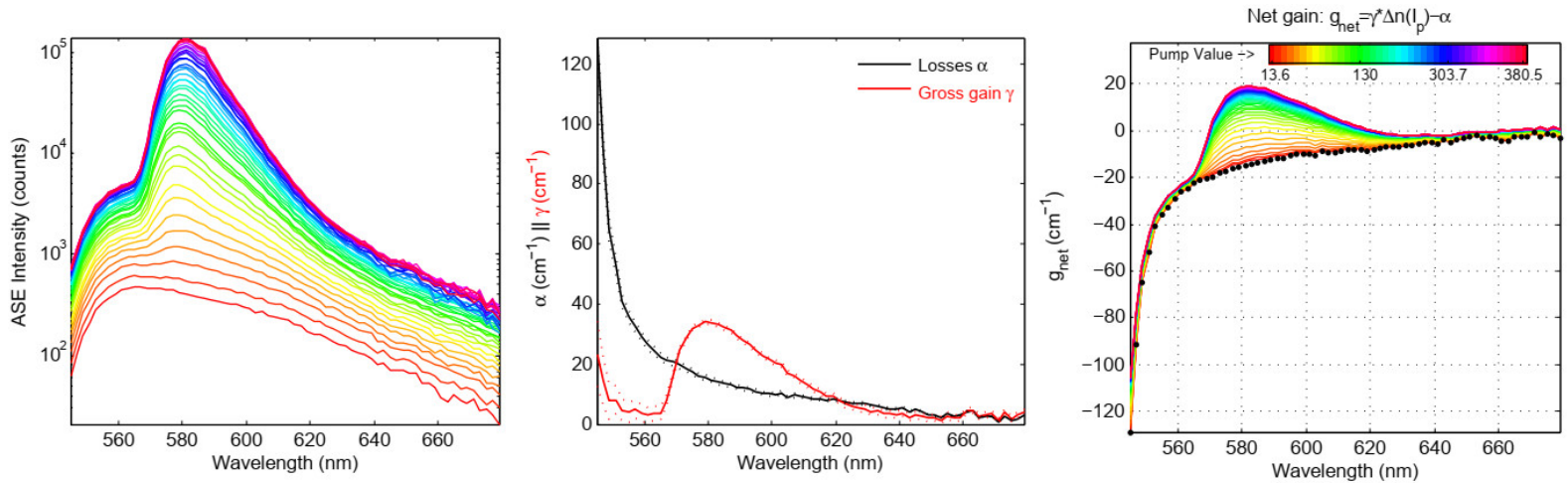

Fig. 1 (Left panel) ASE spectra as a function of pump intensity used for the fit. (Centre panel) Fitted losses $\alpha$ and gross gain $\gamma$ as a function of wavelength. (Right panel) Spectrally resolved net optical gain at increasing pump intensities. Sample: $1 \mu \mathrm{m}$ thick $10 \mathrm{mM}$ Rhodamine 6G (Rh6G) doped pHEMA film deposited onto a quartz substrate.

In this communication, we will revisit the main fundaments of the VPI method and its implementation into the freely distributed software ASE gain Labs, and will highlight the potential of this methodology to unveil relevant information on the photonic properties of the waveguiding devices (mode confinement effects, leakymodes, ...) and on the photophysics of the active materials (excited state absorption, aggregates, ...) [4]. In other words, we will show that the VPI method is a relevant, time-saver, and powerful formalism.

\section{References}

[1] M. Anni, S. Lattante (Eds.), "Organic Lasers: Fundamentals, Developments, and Applications” (Pan Stanford Publ., Singapore, 2018)

[2] L. Cerdán, "Variable Stripe Length method: influence of stripe length choice on measured optical gain," Opt. Lett. 42, 5258-5261 (2017)

[3] L. Cerdán, "Simultaneous retrieval of optical gains, losses, and threshold in active waveguides," Opt. Laser Technol. 121, 105814 (2020)

[4] L. Cerdán, M. Anni, M. L. De Giorgi, P. G. Boj, M. A. Díaz-García, "Probing the limits of the Variable Pump Intensity method to measure optical gains and unveil photophysical and photonic phenomena in waveguides and solutions," submitted. 